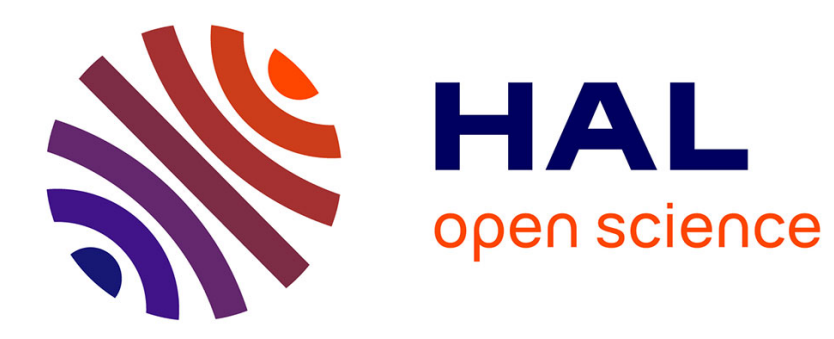

\title{
Weak anchoring effects in smectic-A Fréedericksz transitions
}

Gaetano Napoli, Riccardo de Pascalis

\section{To cite this version:}

Gaetano Napoli, Riccardo de Pascalis. Weak anchoring effects in smectic-A Fréedericksz transitions. 2019. hal-02194075

\section{HAL Id: hal-02194075 \\ https://hal.science/hal-02194075}

Preprint submitted on 25 Jul 2019

HAL is a multi-disciplinary open access archive for the deposit and dissemination of scientific research documents, whether they are published or not. The documents may come from teaching and research institutions in France or abroad, or from public or private research centers.

$$
\text { Copyright }
$$

L'archive ouverte pluridisciplinaire HAL, est destinée au dépôt et à la diffusion de documents scientifiques de niveau recherche, publiés ou non, émanant des établissements d'enseignement et de recherche français ou étrangers, des laboratoires publics ou privés. 


\title{
Weak anchoring effects in smectic- $A$ Fréedericksz transitions
}

\author{
Gaetano Napoli*, Riccardo De Pascalis \\ Dipartimento di Matematica e Fisica 'E. De Giorgi', Università del Salento, \\ Via per Arnesano, 73100, Lecce, Italy
}

\begin{abstract}
In smectic- $A(\mathrm{SmA})$ liquid crystals, rod-like molecules are organized into layers with their optical axis oriented along the normal to these layers. When subject to an external field and for a certain critical threshold, these layers can buckle into a new different configuration. Here, we consider SmA liquid crystals which are confined between two parallel plates aligned in the so-called bookshelf geometry and subject to an external magnetic field. By assuming that instability is induced by a uniform field, we investigate the influence of the anchoring strength on the critical threshold field and on the layers shape by a perturbative analysis to the equilibrium equations. Main differences with respect to the standard Fréedericksz transition of nematics are highlighted. The behaviour of this threshold effect suggests a new way to measure geometrical and constitutive parameters of a SmA sample.
\end{abstract}

KEYWORDS: buckling, instabilties, smectic liquid crystals, layered materials

SUBJECT AREAS: continuum mechanics, condensed soft matter, applied mathematics, mathematical physics

\section{Introduction}

A certain number of condensed phases (cholesteric and smectic liquid crystals [10, diblock copolymers [20, ferrofluids [7, ferrimagnets [26]) can be considered as two-dimensional layered systems with onedimensional translational order. These phases are characterized by a long-range bending and stretching of the layers. They are often confined between two parallel plates and aligned naturally in two configurations: i) with uniform layers parallel to the delimiting plates is the so-called homeotropic alignment; ii) with uniform layers perpendicular to the sample walls named the bookshelf configuration. In order to quantify the material constitutive parameters, both configurations are often used in experiments. Information on the distortion of the layers can be acquired by measuring their optical properties.

Layers of samples in homeotropic alignment might undulate if subject to external magnetic/electric fields. This effect, first observed by Helfrich [11] and Hurault [12] in cholesteric stripes, was then extended and explored in [10, 29, 30, 13, 2, 25, 9, 15]. Similar patterns are obtained also by applying a uniform displacement at one of the two walls [4, 23, 18, 33].

For SmA in a bookshelf configuration, the distortion of the layers induced by external fields is hard to observe since it is unfavourable if compared with the occurrence of topological defects [28]. In fact, the onset of defects causes irreversible distortions, and, in most cases, it happens that no SmA Fréedericksz transition with an elastic distortion analogous to that in the nematic phase is observable. Notwithstanding, elastic distortions have been observed and reported in [8, 21]. As a critical threshold of the applied field is exceeded, distorted layers become energetically favoured with respect to the undeformed ones (see Figure 1 for a schematic representation). In order to predict the critical threshold of this instability, Elston [8] studied the field-induced distortion of the layers of a sample near the smectic- $A$ to smectic- $C$ transition. He derived an expression for the critical voltage which is similar to that found in the case of

\footnotetext{
${ }^{*}$ Author for correspondence: gaetano.napoli@unisalento.it
} 

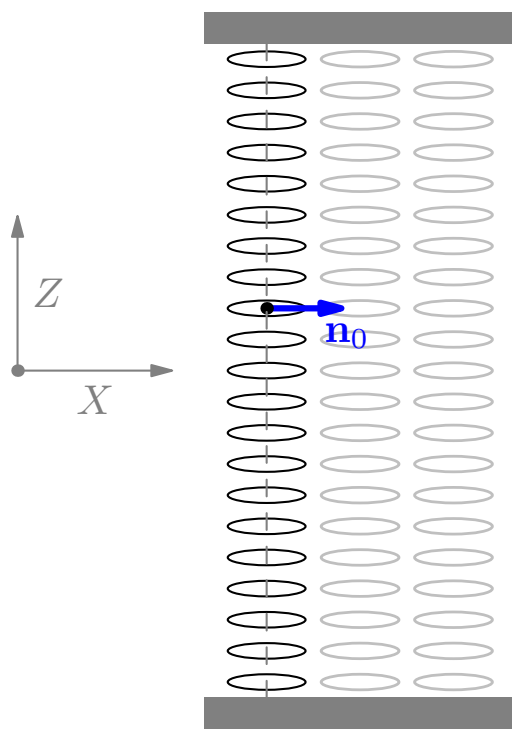

$|\mathbf{H}|<H_{\text {cr }}$
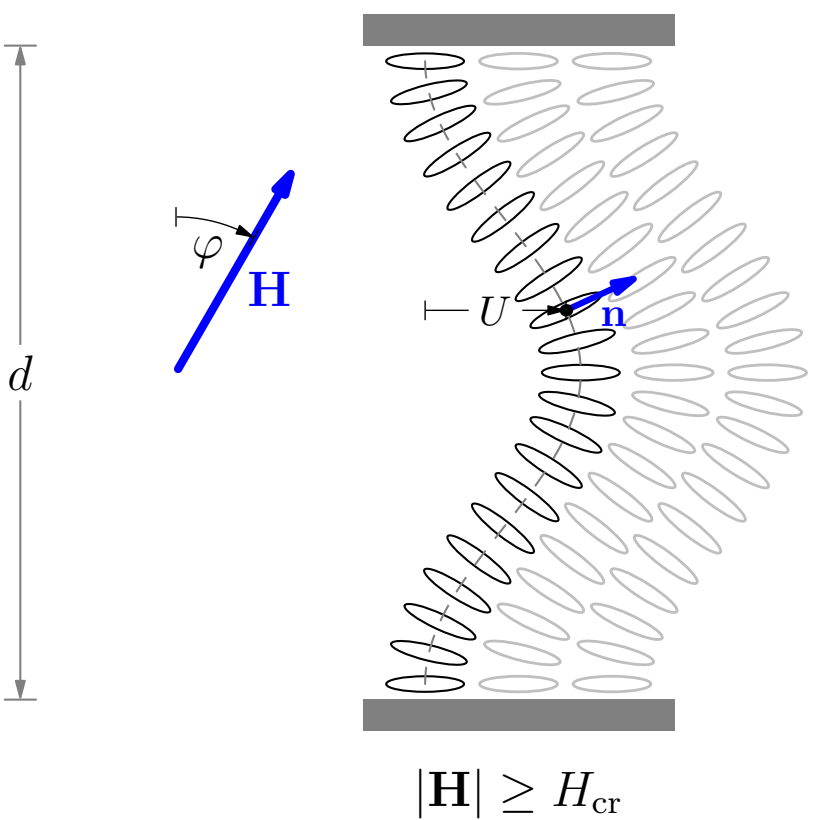

Figure 1: Schematic representation of undeformed lamellar phases in bookshelf configuration (left) and lamellar phases with buckled layers (right).

nematics, but with a threshold voltage twice the one for the nematic Fréedericksz transition. A similar result has been obtained also in [14] for smectic- $C$.

It is well known that the softening of the anchoring conditions produces a lowering of the instability threshold 31. The aim of this paper is to investigate the influence of the anchoring strength on the onset of a Fréedericksz-like instability for SmA ordered in a bookshelf configuration. Whilst the effects due to the weak anchoring have been widely investigated for the nematic phase, there is still a lack of results on smectics. For homeotropic alignements, Ishikawa and Lavrentovich [13] first investigated, both theoretically and experimentally, the effects of a finite anchoring energy on both the critical threshold and layers distortions. Later, De Vita and Stewart [5] explored the influence of weak anchoring upon the alignment of SmA liquid crystals with surface pretilt. In both cases, the weak anchoring surface energy density has been taken in the form dictated by the Rapini-Papoular formula [22].

Here, we also consider an anchoring potential as in [22]. However, unlike the model used in [5] and [8, we assume that the liquid crystal optical axis is perfectly aligned with the normal to the layers [32, 24, 25, 18. This assumption, imposed as a kinematical constraint, holds well below the smectic- $A$ to smectic- $C$ transition temperature [10], where the energetic cost of the layers distortion is small if compared to that of the tilt of the optical axis with respect to the normal to the layers. Moreover, the same assumption allows to extend this model to other lamellar phases such as cholesteric stripes [12, 13].

The above kinematical constraint, on one hand, offers the advantage to reduce the degrees of freedom of the problem, but on the other hand, it entails that the distortion energy involves the layers curvature. Consequently, the energy functional, which has to be minimized, becomes an higher-order Lagrangian. Indeed, the related Euler-Lagrange equation is a fourth-order ordinary differential equation, where the unknown is the layers displacement. Moreover, the presence of the anchoring potential leads to Robinlike boundary conditions which involve the first and the second derivative of the displacement field.

This paper is organized as follows. In Section II, we illustrate the geometry of the model and we then introduce the energy functional which accounts for the energetic distortion cost: the elastic energy, the field-matter interaction energy and the anchoring potential at the two parallel infinite delimiting plates. The Euler-Lagrange equations are derived in Appendix A. In Sect. III, in order to compute the critical threshold and the shape of the layers, we consider the linearized version of the obtained boundary value problem. We study the onset of the instability by varying the anchoring strength and the magnetic field alignment. Some conclusions are offered in section IV. 


\section{The model}

\subsection{Geometric preliminaries}

Let $O(X, Y, Z)$ be a Cartesian coordinate system in the undeformed state with the $X$-axis parallel to the unit normal vector $\mathbf{n}^{0}$ and $O(x, y, z)$ a Cartesian frame in the deformed configuration. Smectic- $A$ liquid crystals can be described by isosurfaces $\mathcal{S}_{k}$ ( $k$ labels the layer) defined by

$$
\omega(\mathbf{x}, k)=0,
$$

where $\mathbf{x}$ denotes the position of a point on $\mathcal{S}_{k}$. In particular, in the undeformed state such surfaces are parallel planes represented by

$$
\omega_{0}(\mathbf{X}, k)=\mathbf{n}_{0} \cdot \mathbf{X}-k \ell_{0}, \quad \mathbf{X}=X \mathbf{e}_{1}+Z \mathbf{e}_{3},
$$

where $\ell_{0}$ represents the distance between two adjacent planes.

Let us consider the invertible transformation $\mathbf{x}=\chi(\mathbf{X})$, which maps the undeformed configuration into the distorted one. By using its inverse $\mathbf{X}=\chi^{-1}(\mathbf{x})$, equation (2) yields

$$
\omega(\mathbf{x}, k)=\mathbf{n}_{0} \cdot \chi^{-1}(\mathbf{x})-k \ell_{0} .
$$

By taking the spatial gradient of equation (1) and by application of the chain rule, we obtain

$$
\nabla \omega=\frac{\partial \omega}{\partial \mathbf{x}}=\frac{\partial \omega_{0}}{\partial \mathbf{X}} \frac{\partial \mathbf{X}}{\partial \mathbf{x}}=\mathbf{F}^{-\mathrm{T}} \frac{\partial \omega_{0}}{\partial \mathbf{X}}
$$

where $\mathbf{F}$ denotes the deformation gradient, i.e., the Jacobian of the transformation $\chi$, and the superscript $-\mathrm{T}$ denotes the transpose of the inverse.

We consider a cell of material in a bookshelf configuration between two parallel planes $Z=-d / 2$ and $Z=d / 2$ which is subjected to the the following shear deformation

$$
\mathbf{x}=\mathbf{X}+U(Z) \mathbf{e}_{1},
$$

which implies

$$
\mathbf{F}=\mathbf{I}+\frac{\mathrm{d} U}{\mathrm{~d} Z} \mathbf{e}_{1} \otimes \mathbf{e}_{3}, \quad \mathbf{F}^{-\mathrm{T}}=\mathbf{I}-\frac{\mathrm{d} U}{\mathrm{~d} Z} \mathbf{e}_{3} \otimes \mathbf{e}_{1} .
$$

By combining (2) 1 and (4), we obtain

$$
\nabla \omega=\mathbf{F}^{-\mathrm{T}} \mathbf{n}_{0}
$$

We denote $\mathbf{n}$ the director field in the distorted configuration. For smectics- $A$, being $\mathbf{n}$ normal to the layers, we can write

$$
\mathbf{n}=\frac{\nabla \omega}{|\nabla \omega|}=\left(\mathbf{e}_{1}-\frac{\mathrm{d} U}{\mathrm{~d} Z} \mathbf{e}_{3}\right)\left[1+\left(\frac{\mathrm{d} U}{\mathrm{~d} Z}\right)^{2}\right]^{-\frac{1}{2}}
$$

\subsection{Distortion energy density}

When an external field is applied, the layers can undergo a static distortion with respect to their natural configuration. The total energy of the system includes several contributions. Elasticity is accounted by the following elastic energy density [17]

$$
f_{e}=\frac{K}{2}(\operatorname{div} \mathbf{n})^{2}+\frac{\bar{B}}{2}\left(\frac{1}{|\nabla \omega|}-1\right)^{2}
$$

where $K$ and $\bar{B}$ are two positive constants called the bending stiffness and the compression modulus, respectively. Indeed, since $\frac{1}{2} \operatorname{div} \mathbf{n}$ is the mean curvature of the surface representing the layer, the first 
term in (9) penalizes the layer bending. The second term, instead, represents the energy related to the dilation/compression of the layers thickness. The quantity $\lambda=\sqrt{K / \bar{B}}$ defines a characteristic length of the material which is of the order of the layers thickness [10].

By application of the chain rule, we obtain the layers curvature

$$
\operatorname{div} \mathbf{n}=\mathbf{F}^{-\mathrm{T}} \cdot \frac{\partial \mathbf{n}}{\partial \mathbf{X}}=-\frac{\mathrm{d}^{2} U}{\mathrm{~d} Z^{2}}\left[1+\left(\frac{\mathrm{d} U}{\mathrm{~d} Z}\right)^{2}\right]^{-\frac{3}{2}}
$$

and, by using the relation (7), the thickness stretch

$$
\frac{1}{|\nabla \omega|}=\left[1+\left(\frac{\mathrm{d} U}{\mathrm{~d} Z}\right)^{2}\right]^{-\frac{1}{2}}
$$

A first glance at equation (9) reveals that the minimum of the elastic energy is reached at the natural configuration, i.e. for vanishing $U(Z)$.

\subsection{Anchoring potential}

According to the Rapini and Papoular formula [22], we assume that, at the walls, $\mathbf{n}$ prefers to align along the $\mathbf{e}_{1}$ direction. Consequently, the anchoring energy can be written as

$$
f_{a}=\frac{1}{2}\left[w_{-}\left(\mathbf{n} \cdot \mathbf{e}_{3}\right)_{Z=-d / 2}^{2}+w_{+}\left(\mathbf{n} \cdot \mathbf{e}_{3}\right)_{Z=d / 2}^{2}\right]
$$

where $w_{ \pm}$are two positive constants called the anchoring strengths.

\subsection{Field-matter interaction energy density}

According to [6, 10, the interaction density energy between $\mathbf{n}$ and the homogeneous applied magnetic field $\mathbf{H}$ is

$$
f_{i}=-\frac{\chi_{a}}{2}(\mathbf{H} \cdot \mathbf{n})^{2}
$$

where $\chi_{a}$ is the diamagnetic anisotropy. If $\chi_{a}>0$, the interaction energy reaches the minimum when the normal $\mathbf{n}$ is aligned with the field. On the contrary, when $\chi_{a}<0, f_{i}$ reaches the minimum when $\mathbf{n}$ is orthogonal to $\mathbf{H}$.

In the following, we consider the possibility of a misalignment between the applied field and the $Z$-axis (see Figure 1), by

$$
\mathbf{H}=H\left(\sin \varphi \mathbf{e}_{x}+\cos \varphi \mathbf{e}_{z}\right)
$$

which, once plugged into (13), yields

$$
f_{i}=-\frac{\chi_{a}}{2} H^{2}\left(-\frac{\mathrm{d} U}{\mathrm{~d} Z} \cos \varphi+\sin \varphi\right)^{2}\left[1+\left(\frac{\mathrm{d} U}{\mathrm{~d} Z}\right)^{2}\right]^{-1} .
$$

Here, in order to create a competition between the magnetic field and the anchoring strength effects, we assume $\chi_{a}>0$.

\subsection{Non-dimensionalization}

Equilibrium configurations are stationary points of the free energy functional

$$
\mathcal{F}=\int_{-d / 2}^{d / 2}\left(f_{e}+f_{i}\right) \mathrm{d} Z+f_{a}
$$


which satisfies the boundary conditions $U(-d / 2)=U(d / 2)=0$. It is convenient to introduce the dimensionless variables

$$
\zeta=\frac{Z}{d}, \quad u=\frac{U}{d}
$$

as well as the dimensionless magnetic field and anchoring strengths

$$
h=\sqrt{\frac{\chi_{a}}{K}} H d, \quad \beta_{ \pm}=\frac{d}{K} w_{ \pm} .
$$

The quantity $\xi=K / w$, named the extrapolation length, is the measure for the relevance of the competing elastic distortion versus the anchoring induced order. The ratio between the sample thickness $d$ and the extrapolation length measures the strength of the anchoring. Note that strong planar anchoring conditions are recovered in the limit $\beta_{ \pm} \rightarrow \infty$, where layers are clamped at the walls. On the contrary, the conditions $\beta_{ \pm}=0$ express free anchoring conditions and, in this case, the layers are simply supported at the walls.

By using the new variables, the domain is now described by $\zeta$ in $\left[-\frac{1}{2}, \frac{1}{2}\right]$. Let us introduce the following notation: plus and/or minus subscripts denote functions valued at $\zeta=\frac{1}{2}$ and/or $\zeta=-\frac{1}{2}$, respectively. By using (17), (18) and the energy terms defined above, from (16), we obtain the dimensionless energy functional

$$
\begin{aligned}
\mathcal{G} & =\mathcal{F} \frac{d}{K}=\int_{-\frac{1}{2}}^{\frac{1}{2}}\left[\frac{1}{2} \frac{u^{\prime \prime 2}}{\left(1+u^{\prime 2}\right)^{3}}+\frac{1}{2} \frac{d^{2}}{\lambda^{2}}\left(\frac{1}{\sqrt{1+u^{\prime 2}}}-1\right)^{2}\right. \\
- & \left.\frac{h^{2}}{2} \frac{\left(u^{\prime} \cos \varphi+\sin \varphi\right)^{2}}{1+u^{\prime 2}}\right] \mathrm{d} \zeta+\frac{\beta_{+}}{2} \frac{u_{+}^{\prime 2}}{1+u_{+}^{\prime 2}}+\frac{\beta_{-}}{2} \frac{u_{-}^{\prime 2}}{1+u_{-}^{\prime 2}},
\end{aligned}
$$

where prime denotes the differentiation with respect to $\zeta$ and whose stationary points have to satisfy the boundary conditions $u_{-}=u_{+}=0$.

\section{Field-induced distortions}

\subsection{Linearized equilibrium equations}

Let us first consider an applied magnetic field orthogonal to the delimiting walls $(\varphi=0)$ while the case of misaligned field will be considered in the Section 3.3. In order to perform the linear analysis of the problem, we consider here an applied field $h$ and we define the positive perturbative parameter

$$
\epsilon=\sqrt{\frac{h}{h_{0}}-1}
$$

where the dimensionless field $h_{0}$ is the critical threshold at which the layers instability occurs. Then, let us assume that the displacement field can be described by the perturbed function

$$
u(\zeta)=\epsilon v(\zeta)
$$

By replacing equations (20) and (21) into (19) and retaining only the $\mathcal{O}\left(\epsilon^{2}\right)$ terms, we obtain the approximate quadratic functional

$$
\mathcal{G}^{(2)}=\epsilon^{2} \int_{-\frac{1}{2}}^{\frac{1}{2}}\left[\frac{1}{2} v^{\prime \prime 2}-\frac{h_{0}^{2}}{2} v^{\prime 2}\right] \mathrm{d} \zeta+\epsilon^{2} \frac{\beta_{+}}{2} v_{+}^{\prime 2}+\epsilon^{2} \frac{\beta_{-}}{2} v_{-}^{\prime 2} .
$$

The Euler-Lagrange equation (derived in Appendix A) associated to (22) is the fourth-order ordinary differential equation

$$
v^{i v}+h_{0}^{2} v^{\prime \prime}=0
$$


whose solutions must satisfy also the following boundary conditions

$$
\begin{gathered}
v_{ \pm}=0, \\
\pm v_{ \pm}^{\prime \prime}+\beta_{ \pm} v_{ \pm}^{\prime}=0 .
\end{gathered}
$$

Note that conditions (24a) impose fixed layer ends at the walls, while the normal to the layer and the curvature at the wall must satisfy (24b).

In the next Sections, we will analyze the effects of the anchoring strengths on the critical threshold and on the shape of the layers, investigating firstly the symmetric boundary conditions case, i.e. $\beta_{-}=\beta_{+}$ and then the most general asymmetric boundary conditions $\beta_{-} \neq \beta_{+}$.

\subsection{Critical field}

\subsubsection{Symmetric boundary conditions}

In this section, we look for solutions of equation (23) which satisfy boundary conditions (24) when symmetric anchoring strengths $\beta_{+}=\beta_{-}=\beta$ are assumed. The most general solution of equation (23) is

$$
v(\zeta)=\alpha_{1} \cos \left(h_{0} \zeta\right)+\alpha_{2} \sin \left(h_{0} \zeta\right)+\alpha_{3} \zeta+\alpha_{4},
$$

where the coefficients $\alpha_{i}(i=1, . ., 4)$ can be determined by requiring to $u$ to satisfy (24). Conditions (24) lead to a homogeneous system of linear equations, with unknowns $\alpha_{i}$, and nontrivial solutions are admitted if and only if the matrix of the coefficients, we say $M$, is singular. Let us denote $j=\operatorname{det}(M)$, which straightforwardly can be written as $j=2 j_{\mathrm{e}} j_{\mathrm{o}}$, where

$$
\begin{gathered}
j_{\mathrm{e}}=h_{0}^{2} \cos \frac{h_{0}}{2}+h_{0} \beta \sin \frac{h_{0}}{2}, \\
j_{\mathrm{o}}=-h_{0} \beta \cos \frac{h_{0}}{2}+\left(h_{0}^{2}+2 \beta\right) \sin \frac{h_{0}}{2},
\end{gathered}
$$

and where the two subscripts 'e' and 'o' refer to the words 'even' and 'odd', respectively. In fact, in order to obtain nontrivial solutions and by requiring the even symmetry for $v(\zeta)\left(\alpha_{2}=\alpha_{3}=0\right), h_{0}$ and $\beta$ must satisfy the transcendental equation $j_{\mathrm{e}}=0$, which, for $h_{0} \neq 0$, becomes

$$
h_{0} \cot \frac{h_{0}}{2}+\beta=0 \text {, }
$$

while from (25) it holds

$$
v(\zeta)=\alpha_{1}\left(\cos \left(h_{0} \zeta\right)-\cos \frac{h_{0}}{2}\right),
$$

where the coefficient $\alpha_{1}$ is still undetermined. On the contrary, by imposing the odd symmetry $\left(\alpha_{1}=\right.$ $\left.\alpha_{4}=0\right), h_{0}$ and $\beta$ must satisfy $j_{\mathrm{o}}=0$, and (25) reduces now to

$$
v(\zeta)=\alpha_{2}\left[\sin \left(h_{0} \zeta\right)-2 \zeta \sin \left(\frac{h_{0}}{2}\right)\right] .
$$

Note that equation (27) has an analogous for the weak Fréedericksz transition in nematics (see equation (18) in [16]). It admits an infinite (countable) number of solutions for any fixed $\beta$. Thus, apart from the undistorted solution, the linearized equation (23), with imposed boundary conditions (24), admits a family of solutions corresponding to distorted layers and which shape is described by (28), with $h_{0}$ satisfying equation (27). Since opposite applied magnetic fields lead to the same distorted configuration, these solutions are even functions with respect to $h_{0}$. Therefore, without losing of generality, we can assume $h_{0}>0$. 


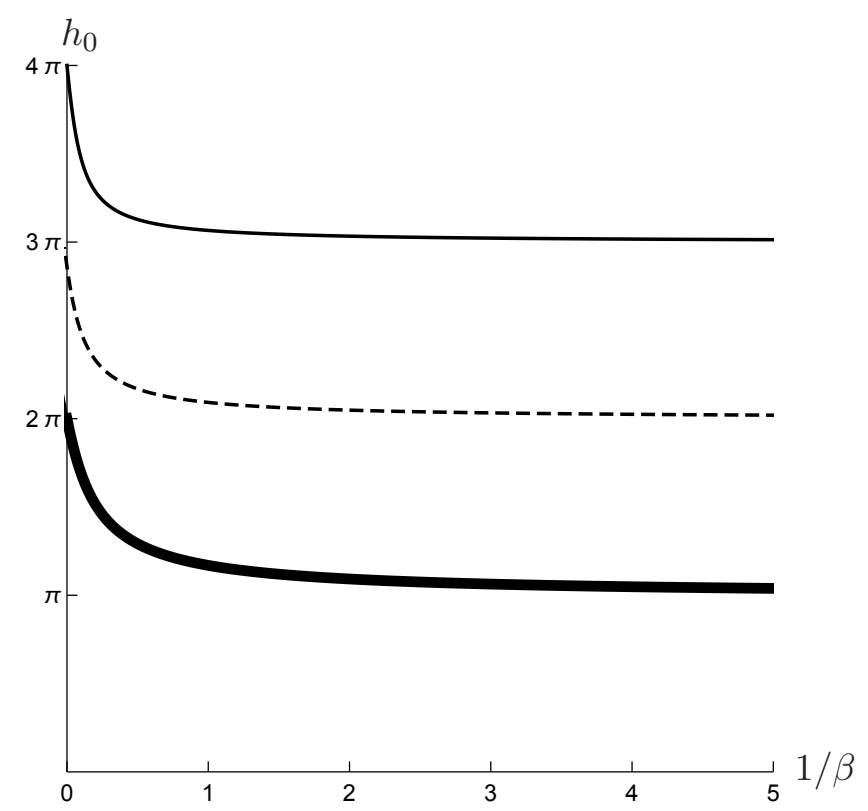

Figure 2: $h_{0}$ plotted as a function of the inverse of the anchoring strength $\beta^{-1}$, according to the equation $j=0$. Solid lines correspond to even solutions $\left(j_{\mathrm{e}}=0\right)$, while the dashed line concerns odd solutions $\left(j_{\mathrm{o}}=0\right)$. The critical field $h_{\mathrm{cr}}=h_{0}$ corresponds to a thicker line.

The critical threshold $h_{\text {cr }}$ is the minimum value of $h_{0}$ for a fixed $\beta$. Hence, $h_{\text {cr }}$ is a solution of (27) lying in the range $[\pi, 2 \pi]$ (in Figure 2 it is represented by a thicker solid line). Thus, the instability with the lowest critical field is the one corresponding to the even symmetry of the layers (28). By analogy with nematics, other symmetries may become locally stable for larger applied field [1, 3].

The infinitely strong planar anchoring conditions are obtained in the limiting case $\beta \rightarrow \infty$. Then, equation (27) yields $h_{\mathrm{cr}}=2 \pi$, while (28) gives

$$
v(z)=\alpha_{1}(\cos (2 \pi z)+1) .
$$

An analogous result is obtained in 27] where the transition is supposed to be induced by the mismatch between the bulk and the surface layer thickness. By using $(\underline{18})_{1}$, we deduce

$$
H_{\mathrm{cr}}=\sqrt{\frac{K}{\chi_{a}}} \frac{2 \pi}{d} .
$$

Likewise in the Fréedericksz transition for nematics, the critical threshold scales as the inverse of the sample thickness $d$. In fact, the equations governing the Fréedericksz transition can be obtained from (23) and (24b) (and disregarding (24a ) ) by replacing $v^{\prime}$ by $\theta$, which is the angle between the nematic director and $\mathbf{e}_{x}$ [16]. However, contrary to the nematic Fréedericksz transition, equation (23) must satisfy the additional boundary conditions (24a) which describes the positional order of the layers on the delimiting walls. As consequence, the symmetry of the solutions is modified. Thus, when layers buckle, the first excited state is the second Fourier mode of $\theta(z)$. On the contrary, in nematics, the director field buckles by involving only the first Fourier mode. This is the reason why, in the limit of strong anchoring, the critical field for smectics- $A$ is twice that for nematics.

The analogous problem for homeotropic alignments has been considered in [2, 11, 13. The transition in this case is named the Helfrich-Hurault effect and the critical field scales with the inverse of the square root of the specimen thickness. Note how the characteristic length $\lambda$ affects the critical field for homeotropic texture, while it does not appear in the expression of critical threshold for bookshelf configurations.

The liming case $\beta \rightarrow 0$ represents the situation where $\mathbf{n}$ can freely rotate at the boundary and, hence, the layers are hinged at the walls. In this latter case, the critical field given by (27) is $h_{\mathrm{cr}}=\pi$ and the solution is of the form $v(\zeta)=\alpha_{1} \cos (\pi \zeta)$. 


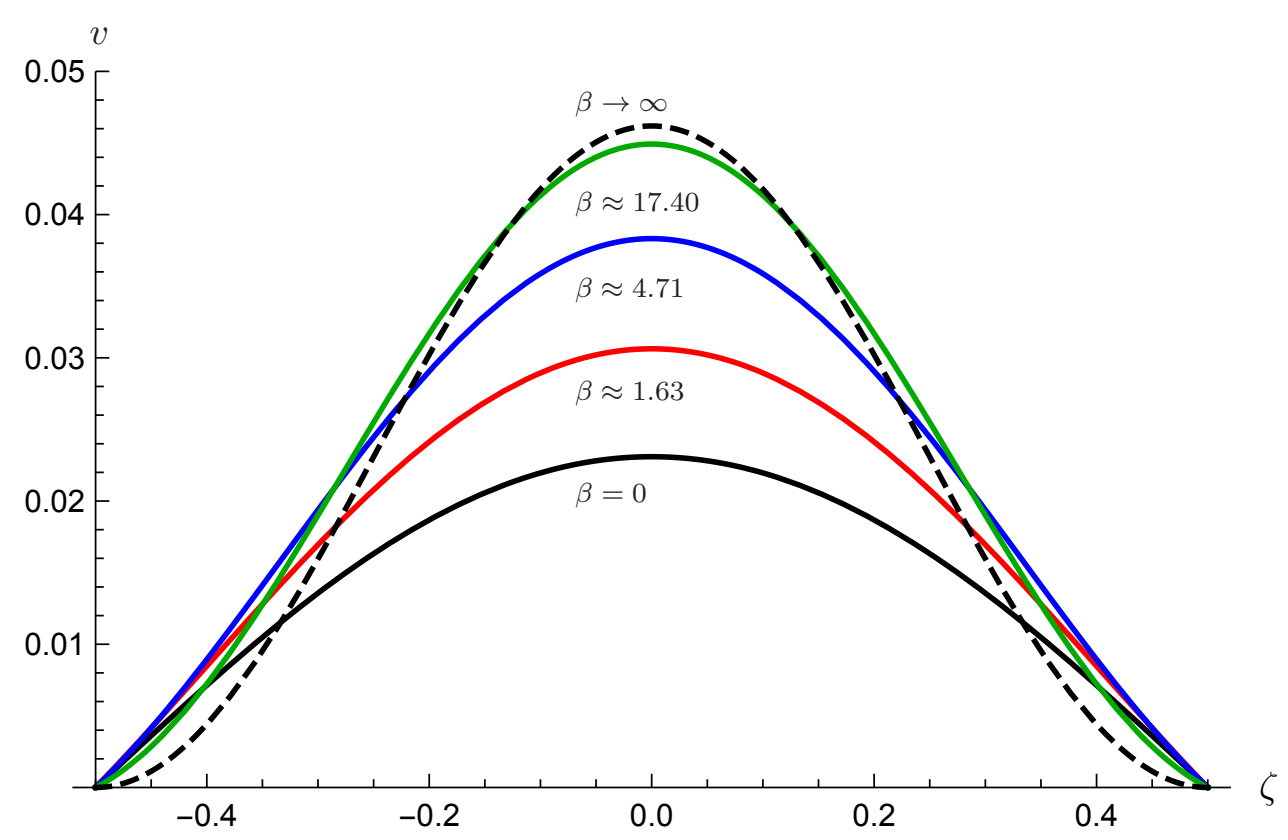

Figure 3: Layers profile at the critical threshold for several values of $\beta$, where $\epsilon=0.1$ and $\lambda / d=0.1$.

Nematics subject to weak planar anchoring boundary conditions exhibit two transitions [16, 31]. The former is the classic Fréedericksz transition, from the planar alignment to the distorted inhomogeneous phase. The latter, referred as the saturation transition, occurs for critical fields larger than the Fréedericksz threshold and describes the switch from the distorted phase to the uniform homeotropically aligned phase. Both the Fréedericksz and the saturation thresholds vanish to zero as anchoring strength vanishes. On the contrary, in smectic phases the saturation is hindered by the presence of the layers. Furthermore, the dimensionless critical field approaches to $\pi$ as anchoring strength vanishes.

\subsubsection{Amplitude determination}

Layers profile is fully understood once the amplitude $\alpha_{1}$ is also determined. Following [19], we considers the fourth-order term in $\epsilon$ of the free energy

$$
\mathcal{G}^{(4)}=\epsilon^{4} \int_{-\frac{1}{2}}^{\frac{1}{2}}\left[-\frac{3}{2}\left(v^{\prime \prime} v^{\prime}\right)^{2}+\frac{1}{8} \frac{d^{2}}{\lambda^{2}} v^{\prime 4}+h_{\mathrm{cr}}^{2} v^{\prime 2}\left(\frac{1}{2} v^{\prime 2}-1\right)\right] \mathrm{d} \zeta-\epsilon^{4} \frac{\beta}{2}\left(v_{-}^{\prime 4}+v_{+}^{\prime 4}\right) .
$$

By replacing (28) into (32) and evaluating the integral, we obtain a $\alpha_{1}$ fourth order polynomial. Then, by minimizing it with respect to $\alpha_{1}$ and by using (27), we obtain two opposite solutions

$$
\alpha_{1}= \pm 4 \sqrt{2} \frac{\lambda}{d} g\left(h_{\mathrm{cr}}\right)
$$

where

$$
g\left(h_{\mathrm{cr}}\right)=\sqrt{\frac{h_{\mathrm{cr}}-\sin h_{\mathrm{cr}}}{6 h_{\mathrm{cr}}-8 \sin h_{\mathrm{cr}}+\sin \left(2 h_{\mathrm{cr}}\right)}},
$$

as well as the trivial solution given by $\alpha_{1}=0$.

In Figure 3 we plotted the function $v$ for some fixed $\beta$. Note that, for larger $\beta$, the layers are clamped (their tangent is horizontal) at the walls $\zeta= \pm \frac{1}{2}$, while they flatten as the anchoring strength vanishes.

\subsubsection{Asymmetric anchoring strength}

Let us consider the case $\beta_{+} \neq \beta_{-}$. In order to study the threshold behavior, we introduce the new variables $\bar{\beta}$ and $\delta$ defined by

$$
\bar{\beta}=\frac{\beta_{+}+\beta_{-}}{2}, \quad \delta=\frac{\beta_{+}-\beta_{-}}{2},
$$




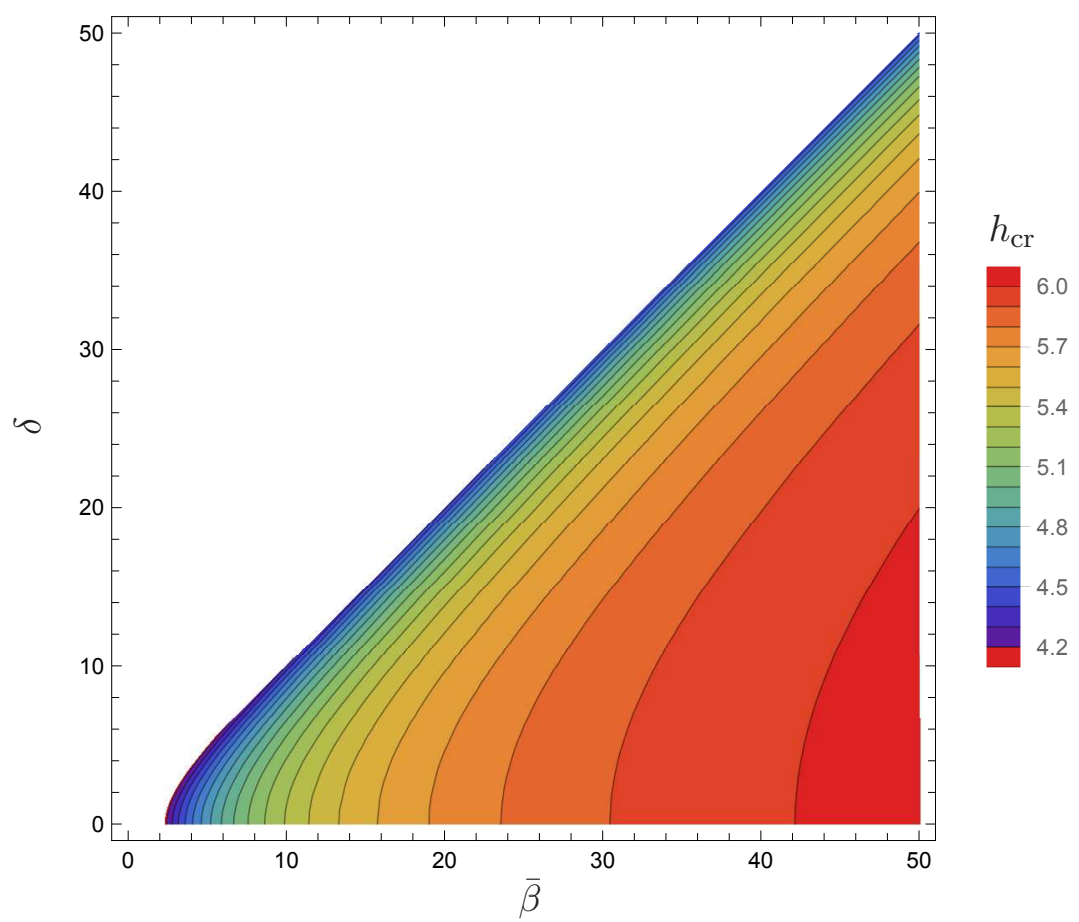

Figure 4: Contour plot of $h_{\mathrm{cr}}(\bar{\beta}, \delta)$ according to equation (36) .

so that $\beta_{ \pm}=\bar{\beta} \pm \delta$. After a tedious but straightforward algebra, from the equation $j=0$, we obtain the general critical equation for the field $h_{\mathrm{cr}}$

$$
2\left(\bar{\beta}^{2}-\delta^{2}\right)+h_{\mathrm{cr}}\left(h_{\mathrm{cr}}^{2}+\delta^{2}-\bar{\beta}^{2}+2 \bar{\beta}\right) \sin h_{\mathrm{cr}}+2\left(\delta^{2}-\bar{\beta}^{2}-h_{\mathrm{cr}}^{2} \bar{\beta}\right) \cos h_{\mathrm{cr}}=0 .
$$

Since both $\beta_{+}$and $\beta_{-}$are positive, without loss of generality, we can assume $\beta_{-} \leq \beta_{+}$, so that $0 \leq \delta \leq \bar{\beta}$. Whilst, $\delta=0$ corresponds to the case previously discussed in previous sections, $\delta=\bar{\beta}$ corresponds to the situation where the layer on the boundary $z=-\frac{1}{2}$ can freely rotate.

For a fixed $\bar{\beta} \geq 0$, the critical threshold $h_{\mathrm{cr}}(\bar{\beta}, \delta)$ determined by (36) is a decreasing monotonic function in $\delta \in[0, \bar{\beta}]$ (see Figure 4 ). The maximum value, achieved in correspondence of symmetric anchoring, is given by equation (27), while the minimum value satisfies the equation

$$
\left(h_{\mathrm{cr}}^{2}+2 \bar{\beta}\right) \sin h_{\mathrm{cr}}-2 h_{\mathrm{cr}} \bar{\beta} \cos h_{\mathrm{cr}}=0,
$$

which is obtained by replacing $\delta=\bar{\beta}$ in equation (36). The solution of (23) which satisfies also the boundary conditions (24a) can be written as

$$
v(\zeta)=\alpha_{1}\left(\cos \left(h_{\mathrm{cr}} \zeta\right)-\cos \frac{h_{\mathrm{cr}}}{2}\right)+\alpha_{2}\left(\sin \left(h_{\mathrm{cr}} \zeta\right)-2 \zeta \sin \frac{h_{\mathrm{cr}}}{2}\right),
$$

while the use of one of the conditions (24b) leads to $\alpha_{2}=\alpha_{1} \delta \nu$, where

$$
\nu=\frac{h_{\mathrm{cr}} \sin \frac{h_{\mathrm{cr}}}{2}}{\beta h_{\mathrm{cr}} \cos \frac{h_{\mathrm{cr}}}{2}-\left(2 \beta+h_{\mathrm{cr}}^{2}\right) \sin \frac{h_{\mathrm{cr}}}{2}},
$$

with $h_{\text {cr }}$ solution of (36).

$\alpha_{2}$ vanishes when the boundary conditions are symmetric, i.e. $\delta=0$, and the layer shape becomes an even function of $\zeta$. Otherwise, the determination of the stationary point of (38) allows to obtain $\Delta$, the $\zeta$-shift of the peak with respect to the centre of the sample. Figure 5 shows the contour plot of $\Delta(\bar{\beta}, \delta)$. Note how the profile peak moves towards the wall where lowest anchoring strength is assumed. Indeed the maximum shift is reached in the case of strong anchoring on one boundary and no-anchoring on the other, and it is expected to be $\Delta_{\max } \approx-0.101$.

Similarly to what we have shown in the previous Section, $\alpha_{1}$ can be determined by using the fourth order free energy expansion. 


\subsection{Misaligned fields}

In this Section, we consider the influence of a misalignment of $\mathbf{H}$ with respect to the $Z$-axis on the Fréedericksz transition. For sake of simplicity, we also assume strong anchoring boundary conditions. By pursuing similar arguments as in the previous Section we obtain the linear equilibrium equation

$$
v^{\prime \prime \prime \prime}+h_{0}^{2} v^{\prime \prime}\left(2 \cos ^{2} \varphi-1\right)=0,
$$

with boundary conditions

$$
v_{ \pm}=0, \quad v_{ \pm}^{\prime}=0 .
$$

Straighforward calculations lead to a critical threshold

$$
h_{\mathrm{cr}}= \pm \frac{2 \pi}{\sqrt{2 \cos ^{2} \varphi-1}} .
$$

Thus, critical field increases (in modulus) with the increasing of the misalignment of the applied field. In particular, $\left|h_{\mathrm{cr}}\right|$ varies in $[2 \pi,+\infty)$ as $|\varphi|$ varies in $[0, \pi / 4]$. Therefore, the instability does not occur when $|\varphi| \geq \pi / 4$.

Similar developments carried in Section 3.2.1 lead to

$$
v(\zeta)=\frac{4 \lambda}{\sqrt{3} d}(\cos (2 \pi \zeta)+1)
$$

which determines completely the profile shape of the layers.

Finally, we can highlight another peculiarity of lamellar phases buckling compared to nematics Fréedericksz transition. In fact, a slight misalignment of the magnetic field is enough to destroy the bifurcation diagram (for further details see $\$ 4.2 .5$ in [31]). Hence, measurements of Frank's constitutive constants for a nematic based on the Fréedericksz effect, is very sensitive to the magnetic field alignment. In smectics, on the contrary, misalignments of the field do not affect the bifurcation diagram, but they modify only the critical threshold (42). However, it is worth to note that the buckling does not occur whenever $|\varphi|$ exceeds $\varphi_{\max }=\pi / 4$.

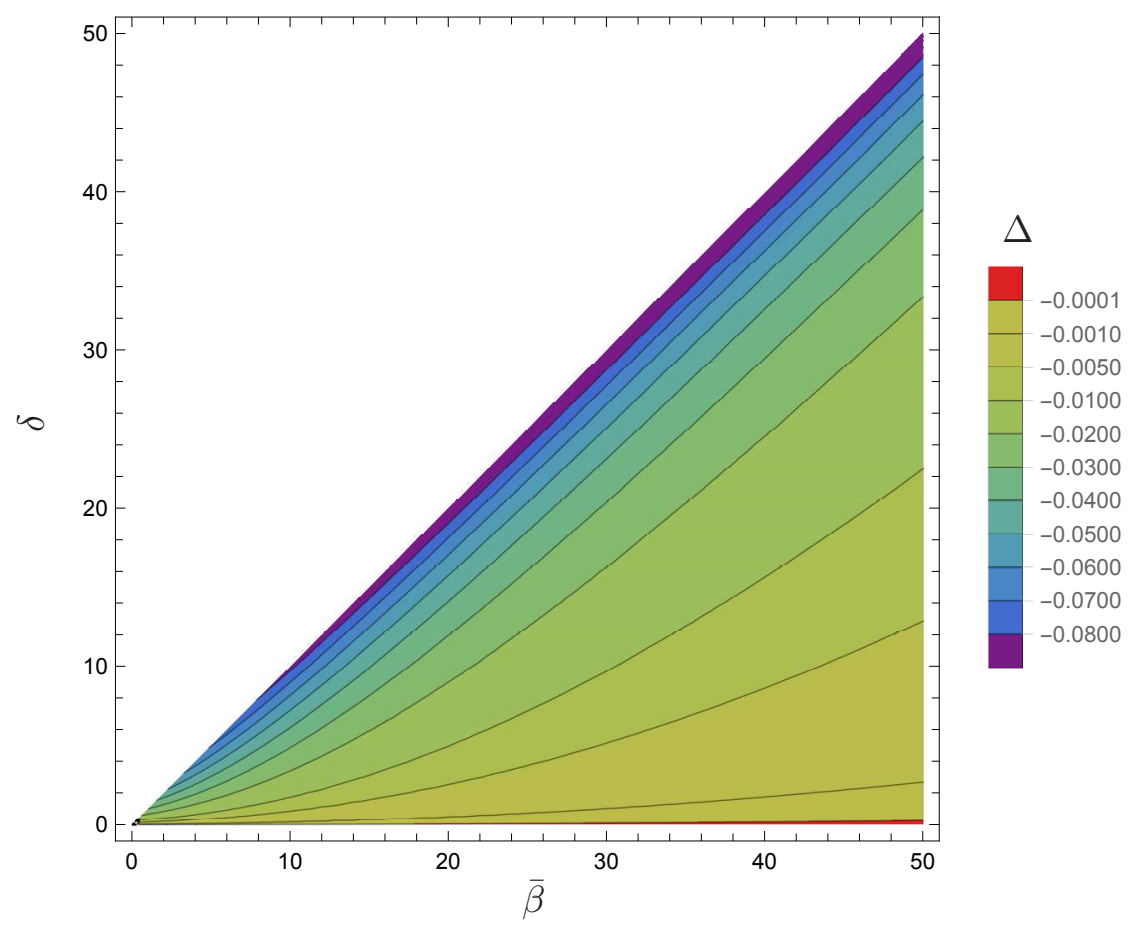

Figure 5: Contour plot of $\Delta(\bar{\beta}, \delta)$. 


\section{Conclusions}

Layers in SmA liquid crystals which are ordered in a bookshelf geometry and subject to a magnetic field can buckle. Here, we have investigated how the onset of layers deformations is affected by the anchoring potential. For symmetric anchoring, i.e. the anchoring strength is the same at the two walls, we obtain a shape profile which is symmetric and the respective critical threshold increases with the anchoring strength. Instead, in the presence of asymmetric anchoring, the maximum displacement moves away from the centre of the cell toward the wall where lowest anchoring strength is imposed.

Furthermore, we analyzed the influence of the misalignment of the magnetic field on the Fréedericksz transition. It emerged that, unlike the Fréedericksz transition for nematics, a misalignment of the magnetic field with respect to the normal to the layer preserves the pitch-fork behavior of the instability. However, this misalignment leads to a raising of the critical threshold that blows up as the misalignment angle reaches $\frac{\pi}{4}$.

Our results could be of particular interest in experiments since allow the theoretical measurement, by inverse analysis, of geometrical and constitutive parameters of the model. For example, by measuring the critical magnetic field and the maximum displacement, the anchoring strength and the characteristic length $\lambda$ can be derived. On the other hand, a difference of anchoring strength between the walls can be detected by measuring the shift of the peak with respect to the centre of the sample.

\section{A Euler-Lagrange equations}

Let $\mathcal{F}$ be a functional with fixed ends $a$ and $b$

$$
\mathcal{F}[u]=\int_{a}^{b} \psi\left(\zeta, u(\zeta), u^{\prime}(\zeta), u^{\prime \prime}(\zeta)\right) \mathrm{d} \zeta+\left.\psi^{a}\left(u(\zeta), u^{\prime}(\zeta)\right)\right|_{\zeta=a}+\left.\psi^{b}\left(u(\zeta), u^{\prime}(\zeta)\right)\right|_{\zeta=b},
$$

and consider a perturbed argument function $u_{\eta}(\zeta)=u(\zeta)+\eta g(\zeta)$ where $\eta$ is a small parameter and $g$ a function which vanishes at the ends. Taylor expansion of $F\left(u_{\eta}, u_{\eta}^{\prime}, u_{\eta}^{\prime \prime}\right)$, up to $\mathcal{O}(\eta)$, yields

$$
\begin{aligned}
& \mathcal{F}\left[u_{\eta}\right]=\int_{a}^{b} \psi\left(u_{\eta}, u_{\eta}^{\prime}, u_{\eta}^{\prime \prime}\right) \mathrm{d} \zeta+\left.\psi^{a}\left(u_{\eta}, u_{\eta}^{\prime}\right)\right|_{\zeta=a}+\left.\psi^{b}\left(u_{\eta}, u_{\eta}^{\prime}\right)\right|_{\zeta=b} \\
&=\int_{a}^{b}\left[\psi\left(u, u^{\prime}, u^{\prime \prime}\right)+\eta\left(\frac{\partial \psi}{\partial u} g+\frac{\partial \psi}{\partial u^{\prime}} g^{\prime}+\frac{\partial \psi}{\partial u^{\prime \prime}} g^{\prime \prime}\right)\right] \mathrm{d} \zeta \\
&+\left[\psi^{a}\left(u, u^{\prime}\right)+\eta \frac{\partial \psi^{a}}{\partial u^{\prime}} g^{\prime}\right]_{\zeta=a}+\left[\psi^{b}\left(u, u^{\prime}\right)+\eta \frac{\partial \psi^{b}}{\partial u^{\prime}} g^{\prime}\right]_{\zeta=b} .
\end{aligned}
$$

From the product differentiation rule we obtain

$$
\begin{gathered}
\frac{\partial \psi}{\partial u^{\prime}} g^{\prime}=\left(g \frac{\partial \psi}{\partial u^{\prime}}\right)^{\prime}-g\left(\frac{\partial \psi}{\partial u^{\prime}}\right)^{\prime} \\
\frac{\partial \psi}{\partial u^{\prime \prime}} g^{\prime \prime}=\left(g^{\prime} \frac{\partial \psi}{\partial u^{\prime \prime}}\right)^{\prime}-\left[g\left(\frac{\partial \psi}{\partial u^{\prime \prime}}\right)^{\prime}\right]^{\prime}+g\left(\frac{\partial \psi}{\partial u^{\prime \prime}}\right)^{\prime \prime} .
\end{gathered}
$$

Thus, by replacing these two expressions into (45), integrating by parts and applying the condition $g(a)=g(b)=0$, we obtain

$$
\begin{aligned}
\mathcal{F}\left[u_{\eta}\right]=\int_{a}^{b} \psi\left(u, u^{\prime}, u^{\prime \prime}\right) \mathrm{d} \zeta+\left[g^{\prime} \frac{\partial \psi}{\partial u^{\prime \prime}}\right]_{a}^{b} \eta & +\int_{a}^{b}\left[\frac{\partial \psi}{\partial u}-\left(\frac{\partial \psi}{\partial u^{\prime}}\right)^{\prime}+\left(\frac{\partial \psi}{\partial u^{\prime \prime}}\right)^{\prime \prime}\right] \eta g \mathrm{~d} \zeta \\
& +\left[\psi^{a}\left(u, u^{\prime}\right)+\eta \frac{\partial \psi^{a}}{\partial u^{\prime}} g^{\prime}\right]_{\zeta=a}+\left[\psi^{b}\left(u, u^{\prime}\right)+\eta \frac{\partial \psi^{b}}{\partial u^{\prime}} g^{\prime}\right]_{\zeta=b}
\end{aligned}
$$

Stationary points of (44) are obtained by imposing

$$
\delta \mathcal{F}=\lim _{\eta \rightarrow 0} \frac{\mathcal{F}\left[u_{\eta}\right]-\mathcal{F}[u]}{\eta}=0 .
$$


By the arbitrariness of $g$ and $g^{\prime}$, the Euler-Lagrange equation

$$
\frac{\partial \psi}{\partial u}-\left(\frac{\partial \psi}{\partial u^{\prime}}\right)^{\prime}+\left(\frac{\partial \psi}{\partial u^{\prime \prime}}\right)^{\prime \prime}=0 \quad \zeta \in(a, b)
$$

and the boundary conditions

$$
\begin{aligned}
& \frac{\partial \psi^{a}}{\partial u^{\prime}}-\frac{\partial \psi}{\partial u^{\prime \prime}}=0 \quad \text { at } \quad \zeta=a, \\
& \frac{\partial \psi^{b}}{\partial u^{\prime}}+\frac{\partial \psi}{\partial u^{\prime \prime}}=0 \quad \text { at } \quad \zeta=b
\end{aligned}
$$

are derived.

\section{References}

[1] Aursand, P., Napoli, G., Ridder, J.: On the dynamics of the weak freedericksz transition for nematic liquid crystals. Communications in Computational Physics 20(5), 1359-1380 (2016)

[2] Bevilacqua, G., Napoli, G.: Reexamination of the helfrich-hurault effect in smectic-a liquid cryst als. Phys. Rev. E 72(4), 041708 (2005)

[3] Bevilacqua, G., Napoli, G.: Parity of the weak fréedericksz transitionedericksz transition. Eur. Phys. J. E 35(12), 133 (2012)

[4] Clark, N.A., Meyer, R.B.: Strain-induced instability of monodomain smectic $a$ and cholesteric liquid crystals. Appl Phys. Lett 22(10), 493-494 (1973)

[5] De Vita, R., Stewart, I.W.: Influence of weak anchoring upon the alignment of smectic a liquid crystals with surface pretilt. Journal of Physics: Condensed Matter 20(33), 335101 (2008)

[6] Deuling., H.: Deformation of nematic liquid crystals in an electric field. Mol. Cryst. Liq. Cryst. 19, $123(1972)$

[7] Elias, F., Flament, C., Bacri, J.C., Neveau, S.: Macro-organized patterns in ferrofluid layer: Experimental studies. J. Phis. I 7, 711 (1997)

[8] Elston, S.J.: Smectic-A Fréedericksz transition. Phy. Rev. E 58(2), R1215-R1217 (1998)

[9] García-Cervera, C.J., Joo, S.: Analytic description of layer undulations in smectic a liquid crystals. Arch. Rat. Mech. Anal. 203(1), 1-43 (2012)

[10] de Gennes, P., Prost, J.: The Physics of Liquid Crystals, 2nd edn. Clarendon Press, Oxford (1993)

[11] Helfrich, W.: Deformation of cholesteric liquid crystals with low threshold voltage. Appl. Phys. Lett 17(12), 531-532 (1970)

[12] Hurault, J.: Static distortions of a cholesteric planar structure induced by magnet ic or ac electric fields. J. Chem. Phys. 59(4), 2068-2075 (1973)

[13] Ishikawa, T., Lavrentovich, O.D.: Undulations in a confined lamellar system with surface anchoring. Phys. Rev. E 63(3), 030501 (2001)

[14] Kedney, P.J., Stewart, I.W.: The onset of layer deformations in non-chiral smectic C liquid crystals. Zeitschrift für angewandte Mathematik und Physik ZAMP 45(6), 882-898 (1994)

[15] Mirantsev, L.V.: Dynamics of helfrich-hurault deformations in smectic-a liquid crystals. Eur. Phys. J. E 38(9), 104 (2015) 
[16] Napoli, G.: Weak anchoring effects in electrically driven freedericksz transitions. J. Phys. A: Math. Gen. 39, 11-31 (2005)

[17] Napoli, G.: On smectic-a liquid crystals in an electrostatic field. IMA Journal of Applied Mathematics 71(1), 34-46 (2006)

[18] Napoli, G., Nobili, A.: Mechanically induced helfrich-hurault effect in lamellar systems. Phys. Rev. E 80(3), 031710- (2009)

[19] Napoli, G., Turzi, S.: On the determination of nontrivial equilibrium configurations close to a bifurcation point. Computers \& Mathematics with Applications 55(2), 299-306 (2008)

[20] Onuki, A., Fukuda, J.I.: Electric field effects and form birefringence in diblock copolymers. Macromolecules 28, 8788 (1996)

[21] Poursamad, J.B., Hallaji, T.: Freedericksz transition in smectic-a liquid crystals doped by ferroelectric nanoparticles. Physica B: Condensed Matter 504, 112-115 (2017)

[22] Rapini, A., Papoular., M.: Distortion d'une lamelle nématique sous champ magnétique. conditions d'angrage aux paroix. J. Phys. Colloque C4 p. 54 (1969)

[23] Ribotta, R., Durand, G.: Mechanical instabilities of smectic- $a$ liquid crystals under dilatative or compressive stresses. J. Phys 38, 179-203 (1977)

[24] Santangelo, C.D., Kamien, R.D.: Curvature and topology in smectic-a liquid crystals. Proceedings of the Royal Society A: Mathematical, Physical and Engineering Science 461(2061), 2911-2921 (2005)

[25] Senyuk, B.I., Smalyukh, I.I., Lavrentovich, O.D.: Undulations of lamellar liquid crystals in cells with finite surface anchoring near and well above the threshold. Phys. Rev. E 74(1) (2006)

[26] Seul, M., Wolfe, R.: Evolution of disorder in magnetic stripe domains. i. transverse instabilities and disclination unbinding in lamellar patterns. Phys. Rev. A 46(12), 7519-7533 (1992)

[27] Shalaginov, A.N., Hazelwood, L.D., Sluckin, T.J.: Dynamics of chevron structure formation. Phys. Rev. E 58(6), 7455-7464 (1998)

[28] Siemianowski, S., Brimicombe, P., Jaradat, S., Thompson, P., Bras, W., Gleeson, H.: Reorientation mechanisms in smectic a liquid crystals. Liquid Crystals 39(10), 1261-1275 (2012). DOI 10.1080/ 02678292.2012.714486. URL https://doi.org/10.1080/02678292.2012.714486

[29] Singer, S.J.: Layer buckling in smectic-A liquid crystals and two-dimensional stripe phases. Phys. Rev. E 48(4), 2796-2804 (1993)

[30] Stewart, I.W.: Layer undulations in finite samples of smectic-A liquid crystals subjected to uniform pressure and magnetic fields. Phys. Rev. E 58(5), 5926-5933 (1998)

[31] Virga, E.G.: Variational theories for liquid crystals. Chapman \& Hall (1993)

[32] Weinan, E.: Nonlinear continuum theory of smectic-A liquid crystals. Arch Rat Mech Anal 137(2), $159-175(1997)$

[33] De Pascalis, R.: Mechanically induced Helfrich-Hurault effect in a confined lamellar system with finite surface anchoring. Phys. Rev. E 100(1), 012705 (2019) 